Contrasting effects of nitric oxide and corticotropin- releasing factor within the dorsal periaqueductal gray on defensive behavior and nociception in mice

T.T. Miguel, K.S. Gomes and R.L. Nunes-de-Souza

The Brazilian Journal of Medical and Biological Research is partially financed by

\section{QR CNPq}

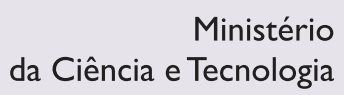

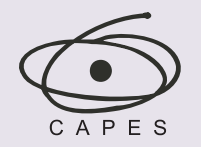

Ministério da Educação
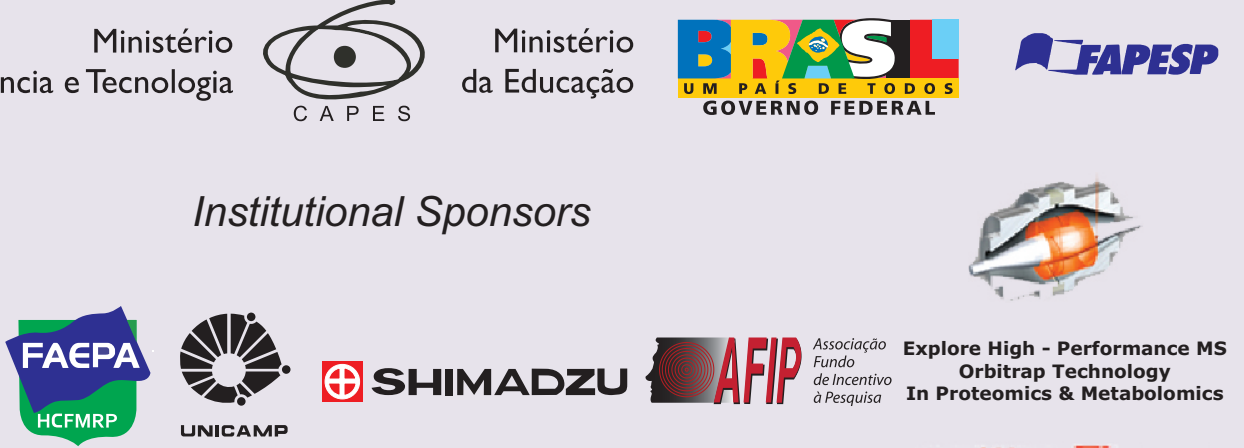

Institutional Sponsors

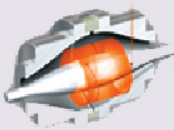

Ф SHIMADZU UNICAMP

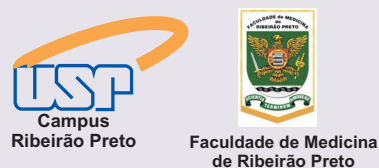

de Ribeirão Preto
de 


\title{
Contrasting effects of nitric oxide and corticotropin- releasing factor within the dorsal periaqueductal gray on defensive behavior and nociception in mice
}

\author{
T.T. Miguel ${ }^{1,2}$, K.S. Gomes ${ }^{2}$ and R.L. Nunes-de-Souza ${ }^{1,2}$ \\ ${ }^{1}$ Programa Interinstitucional de Pós-Graduação em Ciências Fisiológicas, Universidade Federal de São Carlos and \\ Universidade Estadual Paulista, Araraquara, SP, Brasil \\ 2Laboratório de Farmacologia, Faculdade de Ciências Farmacêuticas, \\ Universidade Estadual Paulista, Araraquara, SP, Brasil
}

\begin{abstract}
The anxiogenic and antinociceptive effects produced by glutamate $\mathrm{N}$-methyl-D-aspartate receptor activation within the dorsal periaqueductal gray (dPAG) matter have been related to nitric oxide (NO) production, since injection of NO synthase (NOS) inhibitors reverses these effects. dPAG corticotropin-releasing factor receptor (CRFr) activation also induces anxiety-like behavior and antinociception, which, in turn, are selectively blocked by local infusion of the CRF type 1 receptor (CRFr1) antagonist, NBI 27914 [5-chloro-4-(N-(cyclopropyl)methyl-N-propylamino)-2-methyl-6-(2,4,6-trichlorophenyl)aminopyridine]. Here, we determined whether i) the blockade of the dPAG by CRFr1 attenuates the anxiogenic/antinociceptive effects induced by local infusion of the NO donor, NOC-9 [6-(2-hydroxy-1-methyl-2-nitrosohydrazino)-N-methyl-1-hexanamine], and ii) the anxiogenic/antinociceptive effects induced by intra-dPAG CRF are prevented by local infusion of $\mathrm{N}^{\omega}$-propyl-L-arginine (NPLA), a neuronal NOS inhibitor, in mice. Male Swiss mice (12 weeks old, $25-35 \mathrm{~g}, \mathrm{~N}=8$-14/group) were stereotaxically implanted with a 7-mm cannula aimed at the dPAG. Intra-dPAG NOC-9 (75 nmol) produced defensive-like behavior (jumping and running) and antinociception (assessed by the formalin test). Both effects were reversed by prior local infusion of NBI 27914 (2 nmol). Conversely, intra-dPAG NPLA $(0.4 \mathrm{nmol})$ did not modify the anxiogenic/antinociceptive effects of CRF (150 pmol). These results suggest that CRFr1 plays an important role in the defensive behavior and antinociception produced by NO within the dPAG. In contrast, the anxiogenic and antinociceptive effects produced by intra-dPAG CRF are not related to NO synthesis in this limbic midbrain structure.
\end{abstract}

Key words: Nitric oxide; Corticotropin-releasing factor; Periaqueductal gray; Defensive behavior; Antinociception; Mice

\section{Introduction}

Electrical or chemical stimulation of the dorsal portion of the midbrain periaqueductal gray (dPAG) matter in rats and mice induces vigorous flight and vertical jumping alternating with freezing behavior $(1,2)$. These aversive responses are quite similar to escape reactions elicited by natural predators (3) and, in general, such a defensive behavioral profile is accompanied by antinociception (4). This type of environmentally induced pain inhibition has been suggested to be an adaptive response of animals to cope with situations of imminent danger (4).

A broad range of neurotransmitters released into the PAG have been shown to play important roles in the mediation of defensive behaviors. For instance, it has been shown that serotonin, gamma-aminobutyric acid (GABA), glutamate and neuropeptides such as opioid and corticotropin-releasing factor
(CRF) receptor agonists and antagonists change defensive behavior when injected into this midbrain structure $(5,6)$.

As a ubiquitous excitatory amino acid in the CNS, glutamate activates the ionotropic N-methyl-D-aspartate receptor (NMDAr), as well as two other ion channel-coupled receptors, the a-amino-3-hydroxy-5-methyl-4-isoxazole propionic acid (AMPA) receptor and kainate receptor, and some metabotropic receptor subtypes, the G-protein-coupled receptors (7). The NMDAr is largely expressed in the PAG and its activation with exogenous NMDA elicits fight/flight reactions and antinociception in rats (8) and mice (1). NMDAr activation leads to calcium influx into the cell, which triggers a cascade of intracellular events, including activation of nitric oxide synthase (NOS), an enzyme that produces nitric oxide (NO) by conversion of L-

Correspondence: R.L. Nunes-de-Souza, Laboratório de Farmacologia, Faculdade de Ciências Farmacêuticas, UNESP,

Rodovia Araraquara-Jaú, km 1, 14801-902 Araraquara, SP, Brasil. Fax: +55-16-3301980. E-mail: souzarn@fcfar.unesp.br

Presented at the III Fórum em Neurobiologia do Estresse, Araraquara, SP, Brazil, September 8-10, 2011.

Received December 31, 2011. Accepted March 14, 2012. Available online March 30, 2012. Published April 9, 2012. 
arginine to $\mathrm{L}$-citrulline, using nicotinamide adenine dinucleotide phosphate (NADPH) and $\mathrm{Ca}^{2+}$ as co-factors $(7,9)$. Among the three main NOS isoforms, the neuronal NOS (nNOS) is a constitutive form expressed in neurons (10).

Similarly to the proaversive effects produced by glutamateNMDAr agonists, injection of NO donors into the dPAG produces fight and flight reactions in rats (11). Conversely, intradPAG injection of NOS inhibitors, guanylate cyclase inhibitors and an NO scavenger provokes anxiolytic-like effects in rats exposed to the elevated plus-maze (12). In mice, intra-PAG injection of a highly selective and potent nNOS inhibitor, $\mathrm{N} \omega_{-}$ propyl-L-arginine (NPLA), attenuates defensive behavior in the rat exposure test, a prey-predator interaction test (13), and reverses both the defensive-like behavior and the antinociception induced by local infusion of NMDA (1). These results are consistent with previous findings indicating that there are a large number of NOS immunoreactive neurons in the dPAG of rats and mice $(14,15)$.

Furthermore, it has been shown that NO interferes with the release of several neurotransmitters (e.g., acetylcholine, GABA, glutamate, dopamine, serotonin, and CRF) in distinct brain areas related to the defensive response $(10,16)$. The neuropeptide CRF is strongly linked to stress-related responses, since stress episodes are able to induce CRF release into the brain (17) and to activate the hypothalamic-pituitary-adrenal (HPA) axis (18). CRF-containing neurons and CRF 1 and 2 receptor subtypes (CRFr1 and CRFr2) have usually been identified in the PAG (19). In addition, previous studies have related this neuropeptide to anxiety-and stress-mediated responses, since intracerebroventricular (icv) and intra-dPAG CRF microinjections produce anxiogenic effects $(20,21)$. Moreover, injection of non-selective CRFr antagonists into this midbrain structure prevents anxiety-like behavior elicited by restraint and cold stress and ethanol withdrawal in rats exposed to the elevated plus maze (EPM) (22-24).

We have recently observed that the blockade of CRFr1 with NBI 27914 [5-chloro-4-(N-(cyclopropyl)methyl-N-propylamino)2-methyl-6-(2,4,6-trichlorophenyl)aminopyridine], a potent and selective CRFr1 antagonist (25), within the mouse dPAG abolishes the anxiogenic and antinociceptive effects produced by local injection of CRF (25). Thus, considering that intra-dPAG NO donors produce anxiogenic-like effects (11) and that NO diffusion elicits CRF release $(16,26)$, the present study investigated whether the blockade of CRFr1 would attenuate the anxiogenic and antinociceptive effects of NO donors within this midbrain structure (Experiment 1). In addition, we attempted to show whether the anxiogenic and antinociceptive effects produced by intra-dPAG CRF are attenuated by prior local injection of the nNOS inhibitor NPLA (Experiment 2). Given that intra-dPAG injection of NOC-9 [6-(2-hydroxy-1-methyl-2nitrosohydrazino)-N-methyl-1-hexanamine] provokes explosive defensive behavior, the behavioral test was carried out in a glass cage (Experiment 1). Although intra-dPAG infusion of CRF also increases defensive behavior, this reaction is not so extreme; thus, the behavioral test used in Experiment 2 was reliably assessed with the EPM.

\section{Material and Methods}

\section{Subjects}

Male adult Swiss mice (Universidade Estadual Paulista, UNESP, Brazil) weighing 25-35 $\mathrm{g}$ at testing were used in the study. Mice were housed in groups of 10 per cage $(41 \times 34 \mathrm{x}$ $16 \mathrm{~cm}$ ) and maintained under a normal 12-h light-dark cycle (lights on at 7:00 am) in a temperature-controlled environment $\left(23 \pm 2^{\circ} \mathrm{C}\right)$. Food and water were freely available except during the brief test periods. All mice were naive at the beginning of the experiments.

\section{Drugs}

Drugs and doses used were as follows: NBI 27914 (Tocris Cookson Inc., USA), a potent and selective (Ki=1.7 nM) CRFr1 antagonist (2 nmol) (25), NOC-9 (Sigma-Aldrich, Brazil), a peroxynitrite production-free NO donor (75 nmol) (11), CRF (150 pmol; Sigma-Aldrich) (25), and NPLA (Tocris Cookson Inc.), a highly selective and potent inhibitor of nNOS (Ki $=57$ $n M)$, which displays 3158- and 149-fold selectivity over iNOS and eNOS, respectively $(0.4 \mathrm{nmol})(1)$. Doses were based on previous studies $(1,11,21,25,27)$. NBI was dissolved in dimethylsulfoxide (70\% DMSO in physiological saline), NOC9 in $1 \mathrm{M}$ Tris solution, $\mathrm{pH} 10$, to prevent NO release before it reaches brain tissue (11) and CRF and NPLA in physiological saline $(0.9 \% \mathrm{NaCl})$.

\section{Surgery and microinjection}

Mice were implanted with a 7-mm stainless steel guide cannula (26 gauge; Insight Equipamentos Científicos Ltda., Brazil) under anesthesia with $80 \mathrm{mg} / \mathrm{kg}$ ketamine plus $8 \mathrm{mg} /$ $\mathrm{kg}$ xylazine (a muscle relaxant). A guide cannula was fixed to the skull using dental acrylic and jeweler's screws. Stereotaxic coordinates, based on the Paxinos and Franklin Atlas (28) for the dPAG, were $4.1 \mathrm{~mm}$ posterior to the bregma, $1.3 \mathrm{~mm}$ lateral to the midline, and $2.2 \mathrm{~mm}$ ventral to the skull surface, with the guide cannula angled $26^{\circ}$ to the vertical and its tip positioned $1 \mathrm{~mm}$ above the target site. A dummy cannula (33 gauge, stainless steel wire; Fishtex Industry and Commerce of Plastics Ltda., Brazil), inserted into each guide cannula immediately after surgery, served to reduce the incidence of occlusion. Postoperative analgesia was provided for 3 days by adding $200 \mathrm{mg} / \mathrm{mL}$ acetaminophen to the drinking water (final concentration $=0.16 \mathrm{mg} / \mathrm{mL}$ ).

Five to 7 days after surgery, the various solutions were injected into the dPAG using microinjection units (33-gauge stainless steel cannulae; Insight Equipamentos Científicos Ltd.), which extended $1.0 \mathrm{~mm}$ beyond the tip of the guide cannula. Each microinjection unit was attached to a 5- $\mu \mathrm{L} \mathrm{Ham-}$ ilton microsyringe via polyethylene tubing (PE-10), and drug administration was controlled by an infusion pump (BI 2000, Insight Equipamentos Científicos Ltd.) programmed to deliver $0.2 \mu \mathrm{L}$ over a period of $30 \mathrm{~s}$. The microinjection procedure 
consisted of gently restraining the animal, removing the dummy cannula and inserting the injection unit, which remained in situ for a further $30 \mathrm{~s}$ after the injection. Successful infusion was confirmed by monitoring the movement of a small air bubble in the PE-10 tubing.

\section{Defensive response analysis}

Immediately after the microinjection procedure (see also General procedure, Experiment 1), each mouse was placed in a glass cage $(30 \times 21 \times 25 \mathrm{~cm})$ to record defensive and exploratory behavior for a 5-min period. The recorded defensive behavior consisted of time spent (in seconds) running [i.e., trotting (running but keeping the same pattern as walking) and galloping (fast running, alternating anterior and posterior limb pairs)] and freezing (complete absence of movement except breathing while the animal exhibits a characteristic tense posture), and frequency of jumps (i.e., upward leaps directed to the wall of the glass cage). The exploratory behavior variables recorded were time of locomotion (i.e., slow walking with elevation of trunk and tail and out of phase stance and swing movements of the contralateral limbs) and frequency of rearing (standing on hind limbs, with both forelimbs off the floor; this measure included both unsupported rearing, and rearing against the wall).

This test was recorded with a camera-TV-DVD system and behavior was subsequently scored by a trained observer.

\section{Elevated plus-maze}

The basic EPM design was closely similar to that originally described by Lister (29) and consisted of two open arms (30 x $5 \times 0.25 \mathrm{~cm})$ and two closed arms $(30 \times 5 \times 15 \mathrm{~cm})$ connected by a common central platform $(5 \times 5 \mathrm{~cm})$. The apparatus was constructed from wood (floor) and transparent glass (clear walls) and was raised to a height of $38.5 \mathrm{~cm}$ above floor level.

After drug administration (see General procedure; Experiment 2) into the dPAG (Figure 1), each mouse was placed in an individual holding cage and then transported to the maze. Testing commenced by placing the subject on the central platform of the maze (facing an open arm), after which the experimenter immediately withdrew to an adjacent laboratory. The videotaped test sessions lasted 5 min and, between subjects, the maze was thoroughly cleaned with $20 \%$ alcohol and dry cloths. All experiments were performed under normal laboratory illumination $(1$ x $60 \mathrm{~W}$ yellow incandescent lamp positioned approximately $1.80 \mathrm{~m}$ above the EPM floor), during the light phase of the light-dark cycle. Videotapes were scored by a trained observer using an ethological analysis package developed by the group of Dr. S. Morato, Faculdade de Filosofia, Ciências e Letras de Ribeirão Preto, USP (Brazil). Behavioral parameters consisted of both conventional spatiotemporal and ethological measures (30). Conventional measures were the frequencies of open- and closed-arm entries (entry = all four paws into an arm) and the time spent in the open arms of the maze. These data were used to calculate the percentage of open-arm entries [(open / total) $x$ 100] and percentage of time spent in each zone of the maze [(time in compartment / $300) \times 100]$. Ethological measures are reported as frequency scores for open-arm end exploration (OAEE = entering the $10-\mathrm{cm}$ distal section of the open arm from the central square), head dipping (HD = exploratory movement of head/shoulders over the side of the maze) and stretched-attend postures (SAP: exploratory posture in which the body is stretched forward then retracted to the original position without any forward locomotion). In view of the importance of the thigmotactic cues for patterns of plus-maze exploration, HD and SAP were further differentiated by recording where on the maze they were displayed. Consistent with earlier reports (30), the closed arms and the central platform were designated "protected" areas together (i.e., offering relative security), while the open arms were designated "unprotected" areas. Data for the HD and SAP measures are thus reported as separate protected and unprotected scores.

\section{Nociception test}

Nociception was assessed by the formalin test (1). The formalin test causes a two-phase nociceptive response (31). The first phase begins immediately after formalin injection and lasts approximately $5 \mathrm{~min}$. It results from the direct stimulation of nociceptors (31). The second phase begins $20 \mathrm{~min}$ after the injection and lasts approximately $40 \mathrm{~min}$. This phase is caused by $\mathrm{C}$-fiber activation (32) and also involves a period of sensitization, during which inflammatory phenomena occur (32). In the present study, $50 \mu \mathrm{L}$ formalin (2.5\% formaldehyde) was injected into the dorsal surface of the right hind paw of the mouse, which was placed in a glass holding cage $(30 \mathrm{x}$ $20 \times 25 \mathrm{~cm}$ ). Given that the second phase of the nociceptive response lasts a long time (about $40 \mathrm{~min}$ ), in the present study we chose this phase of the formalin test to assess the effects of drugs injected into the dPAG.

Therefore, the nociceptive response was recorded by measuring the time (in seconds) spent licking the paw injected with formalin during the second phase, between 25 and $35 \mathrm{~min}$ after injection (a period of $10 \mathrm{~min}$ ).

\section{General procedure}

\section{Experiment 1: effects of combined intra-dPAG injections of NBI 27914 and NOC-9 on defensive and exploratory behavior and nociception in mice}

Defensive and exploratory behavior. Mice received an intradPAG injection of NBI 27914 (pretreatment: 0 or $2 \mathrm{nmol} / 0.2$ $\mu \mathrm{L}$ ) and, $10 \mathrm{~min}$ later, an injection of NOC-9 (treatment: 0 or 75 $\mathrm{nmol} / 0.2 \mu \mathrm{L}$ ) at the same midbrain site. Immediately after the intra-dPAG NOC-9 injection, each mouse was placed in the glass cage to record defensive and exploratory behavior (see details in section "Defensive response analysis").

Nociception test. Formalin $(50 \mu \mathrm{L})$ was injected into the hind paw of mice $48 \mathrm{~h}$ after the evaluation of defensive and exploratory behavior in the glass cage. Fifteen minutes after this injection, each mouse received an intra-dPAG injection 
of NBI 27914 (pretreatment: 0 or $2 \mathrm{nmol} / 0.2 \mu \mathrm{L}$ ) and, $10 \mathrm{~min}$ later, an injection of NOC-9 (treatment: 0 or $75 \mathrm{nmol} / 0.2 \mu \mathrm{L}$ ) at the same midbrain site. Immediately after intra-dPAG NOC-9 injection, mice were placed individually in the glass holding cage for $10 \mathrm{~min}$ (i.e., 25-35 min after the formalin injection) to record the time spent licking the affected paw.

\section{Experiment 2: effects of combined intra-dPAG injections of NPLA and CRF on anxiety-like behavior and nociception in mice}

Anxiety test. Mice received intra-dPAG injection of NPLA (pretreatment: 0 or $0.4 \mathrm{nmol}$ )and, 10 min later, CRF was injected (treatment: 0 or $150 \mathrm{pmol}$ ) into the same midbrain site. Ten minutes later, each mouse was placed on the EPM to record conventional and ethological parameters (for details see section "Defensive response analysis") for a period of $5 \mathrm{~min}$.

Nociception test. Forty-eight hours after the EPM test, formalin was injected into the hind paw of each mouse and, 5 min later, they received an intra-dPAG microinjection of NPLA (pretreatment: 0 or $0.4 \mathrm{nmol}$ ). After a further $10 \mathrm{~min}$, the mice received an injection of CRF ( 0 or $150 \mathrm{pmol}$ ) and, 10 min later, they were placed individually in a glass holding cage for 10 min (i.e., 25-35 min after the formalin injection) to record the time (in seconds) spent licking the affected paw.

Importantly, all animals that had received an intra-dPAG

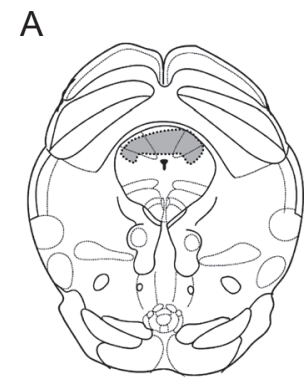

$-4.04$

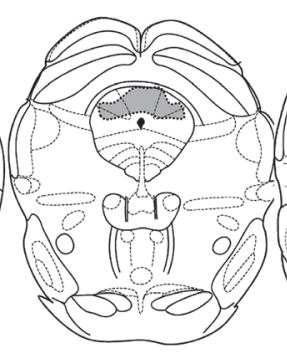

$-4.16$

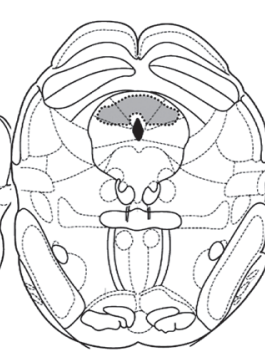

$-4.36$

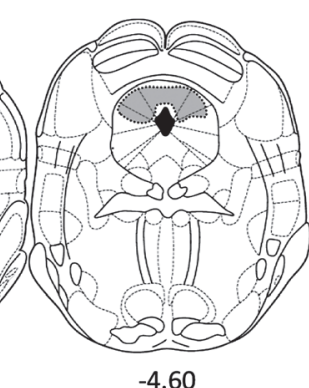

B

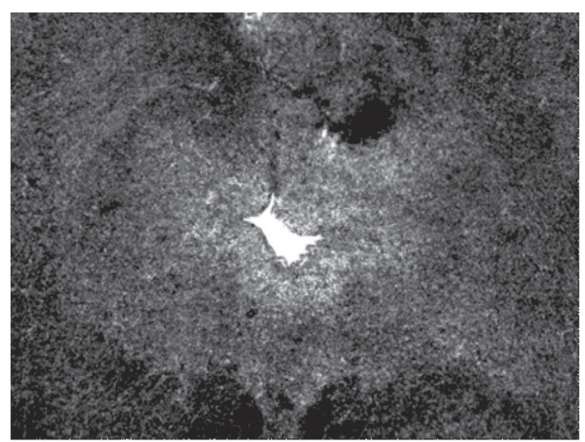

Figure 1. A, Diagram of mouse brain sections, based on Paxinos and Franklin (28), showing microinfusion sites within the midbrain dorsal periaqueductal gray (dPAG). The gray area corresponds to the whole area in which microinjections were placed in the various slices (distance from bregma in $\mathrm{mm}$ ) described in the Paxinos and Franklin Atlas (reproduced with permission). $B$, Photomicrograph of a midbrain coronal section from a representative subject showing an injection site into the dPAG (dark stain: Evan blue). The section was $-4.60 \mathrm{~mm}$ from bregma.
$-4.60$

injection of NOC-9 (75 nmol in Experiment 1) or CRF (150 pmol in Experiment 2) on day 1 (defensive and exploratory behavior and EPM test, respectively) received vehicle on day 3 (nociception test) and vice versa.

\section{Histological analysis}

At the end of testing, all animals received a $0.2-\mu \mathrm{L}$ intradPAG infusion of $1 \%$ Evans blue by the same microinjection procedure as used for the drugs. Animals were then sacrificed in the $\mathrm{CO}_{2}$ chamber, their brains removed and injection sites checked histologically by reference to the Paxinos and Franklin Atlas (28). Data from animals with injection sites outside the dPAG were excluded from data analysis. Final sample sizes ranged from 8 to 11 (defensive and exploratory behaviors) and 8 (nociception test) animals per group in Experiment 1 and from 9 to 14 (anxiety test) and 8 to 11 (nociception test) animals per group in Experiment 2.

\section{Statistical analysis}

All results were initially subjected to Levene's test for homogeneity of variance. Where the Levene test yielded significant inhomogeneity, results were transformed to their log, square root or cube root and confirmed for homogeneity of variance before being subjected to two-way analysis of variance (ANOVA; factor 1 = pretreatment and factor 2 = treatment), followed by the post hoc Duncan test. In those cases (Experiment 1 = running, jumping, and freezing) where the Levene test remained significant even after all transformations, data were analyzed by Kruskal-Wallis non-parametric ANOVA followed by the Dunn test. In all cases, a $P$ value $\leq 0.05$ was required for significance.

\section{Ethics}

The experimental protocols were conducted according to the ethical principles of the Brazilian College of Animal Experimentation (COBEA), and approved by the local Research Ethics Committee (CEP/FCF/Car, Universidade Estadual Paulista, resolution 10/2006).

\section{Results}

\section{Brain injection sites}

Figure 1 shows schematic brain sections based on the Paxinos and Franklin Atlas (28), indicating microinfusion sites within the midbrain dPAG, and a photomicrograph of a midbrain coronal section of a 
representative subject, showing an injection site within the dPAG of the mouse.

\section{Experiment 1: effects of prior intra-dPAG injection of NBI 27914 on the behavior and antinociception} produced by local infusion of NOC-9 at the same site

Figure 2A-E show the effects of intra-dPAG injection of NBI 27914 on the defensive and exploratory behavior produced by local infusion of NOC-9. Kruskal-Wallis ANOVA revealed significant differences in running $(H=23.09$; $P<$ $0.05)$, jumping $(H=20.10 ; P<0.05)$ and freezing $(H=22.06$; $\mathrm{P}<0.05)$ measures. The post hoc Dunn test revealed that intra-dPAG NOC-9 (vehicle + NOC-9) increased the time spent running (2A) and freezing (2C) and the frequency of jumps (2B), relative to the control group (vehicle + vehicle; $\mathrm{P}<0.05)$. Prior intra-dPAG injection of NBI 27914 (NBI + NOC-9) completely blocked the effects of NOC-9 (vehicle + NOC-9) on running and jumping and tended to prevent the freezing induced by the NO donor. Importantly, intradPAG NBI alone (i.e., NBI + vehicle) did not change any behavior. Regarding the exploratory behavior locomotion (Figure 2D), two-way ANOVA revealed significant effects for the pretreatment factor $\left(F_{1,32}=8.29 ; P<0.05\right)$ and treatment factor $\left(F_{1,32}=11.76 ; P<0.05\right)$, but no pretreatment $x$ treatment interaction $\left(F_{1,32}=0.95 ; P=0.34\right)$. Post hoc comparisons revealed that NOC-9 (vehicle + NOC-9) reduced locomotion compared to the control group (vehicle + vehicle). Pretreatment with NBI 27914 reduced the effect of NOC-9 (NBI + NOC-9 vs vehicle + NOC-9; P < 0.05), but did not alter this exploratory behavior when given alone (NBI + vehicle). Two-way ANOVA revealed significant effects regarding rearing frequency (Figure 2E) only for the treatment factor $\left(F_{1,32}=6.35 ; P<0.05\right)$, without showing differences for the pretreatment factor $\left(F_{1,32}=0.26 ; P\right.$ $=0.61)$ or pretreatment $x$ treatment interactions $\left(F_{1,32}=\right.$ $0.006 ; P=0.94)$. The post hoc Duncan test revealed that intra-dPAG NOC-9 reduced the rearing frequency of the control group (vehicle + vehicle).

Figure 3 shows the effects of intra-dPAG injection of NBI 27914 on the antinociceptive effect produced by local infusion of NOC-9. Two-way ANOVA revealed significant differences for the pretreatment factor $\left(\mathrm{F}_{1,28}=4.05\right.$; $\mathrm{P}<$ $0.05)$, treatment factor $\left(F_{1,28}=10.60 ; P<0.05\right)$ and pretreatment $x$ treatment interaction $\left(F_{1,28}=5.12 ; \mathrm{P}<0.05\right)$. The post hoc Duncan test showed that NOC-9 produced antinociception (vehicle + NOC-9 vs vehicle + vehicle) while
A

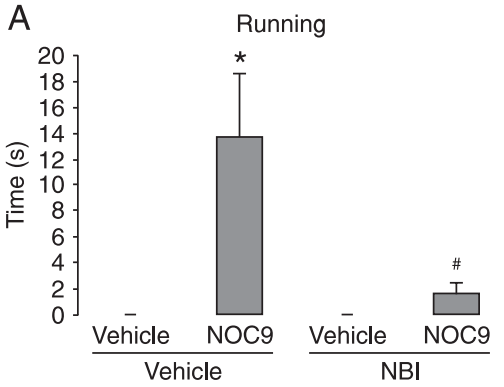

Defensive behaviors
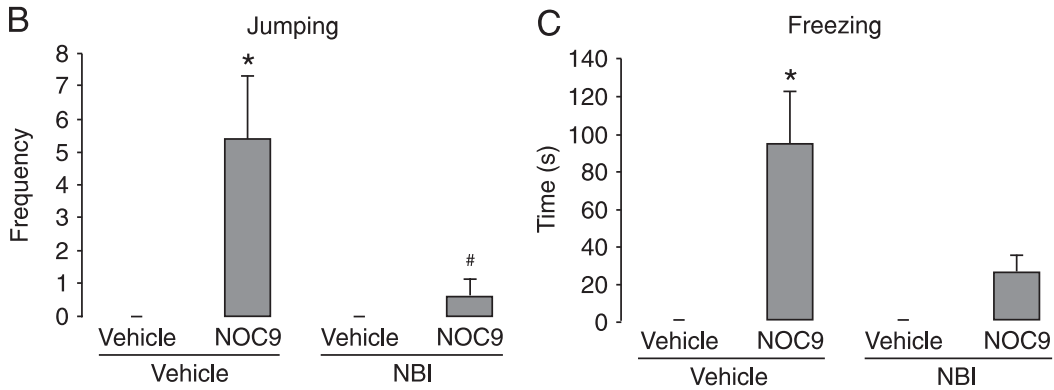

Exploratory behaviors
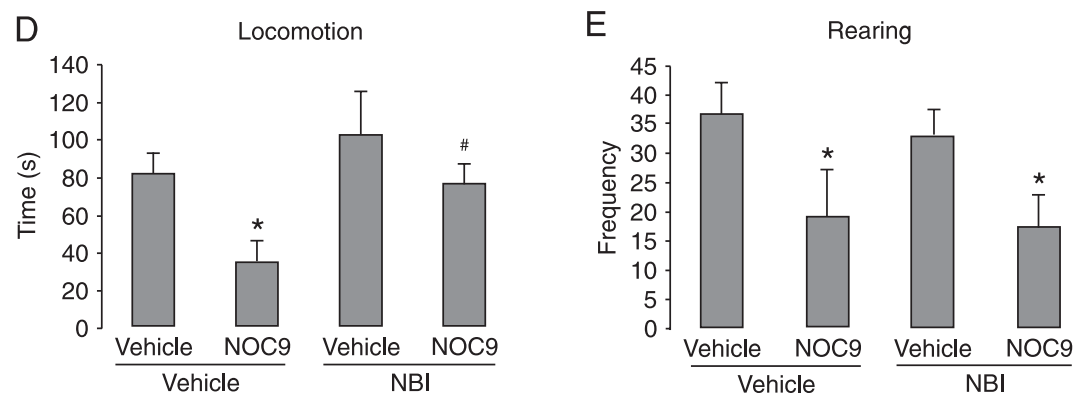

Figure 2. Effect of intra-dPAG microinjections of NBI 27914 (0 or $2 \mathrm{nmol}$ ) and NOC-9 (0 or $75 \mathrm{nmol})$ upon defensive [running $(A)$, jumping $(B)$, and freezing $(C)$ ] and exploratory [locomotion $(D)$ and rearing $(E)]$ behavior of mice. Mice received an intra-dPAG injection of NBI 27914 (pretreatment: 0 or $2 \mathrm{nmol} / 0.2 \mu \mathrm{L}$ ) and, 10 min later, an injection of NOC-9 (treatment: 0 or $75 \mathrm{nmol} / 0.2 \mu \mathrm{L}$ ) at the same midbrain site and were then tested immediately. Data are reported as means $\pm S E M, N=8-11$ per group. dPAG $=$ dorsal periaqueductal gray; NBI 27914 = [5-chloro-4-(N-(cyclopropyl)methyl-N-propylamino)-2-methyl-6-(2,4,6-trichlorophenyl)aminopyridine]; NOC-9 $=$ [6-(2-hydroxy-1-methyl-2-nitrosohydrazino)- $\mathrm{N}$-methyl-1-hexanamine]. ${ }^{*} \mathrm{P}<0.05$ compared to control group (vehicle + vehicle), and ${ }^{\#} \mathrm{P}<0.05$ compared to vehicle + NOC-9 group (Kruskal-Wallis followed by the Dunn post hoc test or two-way ANOVA followed by the Duncan post hoc test). 
NBI 27914 pretreatment completely blocked this effect of NOC-9, without changing the nociceptive response when given alone (NBI + vehicle).

\section{Experiment 2: effects of combined intra-dPAG injections of NPLA and CRF on anxiety-like behavior and nociception in mice}

Figure $4 A$ shows the effects of intra-dPAG injections of first NPLA ( 0 or $0.4 \mathrm{nmol}$ ) and then CRF ( 0 or $150 \mathrm{pmol}$ ) on the behavior of mice exposed to the EPM. Two-way ANOVA revealed significant effects of the treatment (CRF) factor for both indices of anxiety (\%OE, open-arm entry: $F_{1,38}=14.53$; $\mathrm{P}<0.05$; \%OT, open-arm time: $\mathrm{F}_{1,38}=9.92$; $\left.\mathrm{P}<0.05\right)$ but did not show any effect for the pretreatment (NPLA) factor (\%OE: $F_{1,38}=0.06 ; P=0.81 ; \% O T: F_{1,38}=0.03 ; P=0.85$ ) or pretreatment $x$ treatment interaction $\left(\% O E: F_{1,38}=0.48\right.$; $\left.\mathrm{P}=0.49 ; \% \mathrm{OT}: \mathrm{F}_{1,38}=1.42 ; \mathrm{P}=0.24\right)$. Post hoc analysis revealed that CRF (vehicle $+\mathrm{CRF}$ ) decreased both indices of anxiety (\%OE and \%OT, $\mathrm{P}<0.05)$ compared to the control group (vehicle + vehicle), and these effects were not blocked by prior intra-dPAG injection of NPLA. In addition, one-way ANOVA did not reveal any effect of the pretreatment factor $\left(F_{1,38}=0.016 ; P=0.90\right)$, treatment factor $\left(F_{1,38}=0.005\right.$; $\mathrm{P}=0.94)$ or pretreatment $\mathrm{x}$ treatment interaction $\left(\mathrm{F}_{1,38}=\right.$ $0.67 ; \mathrm{P}=0.42$ ) on closed-arm entries.

As shown in Figure 4B, ANOVA revealed some significant effects of CRF treatment on ethological measures

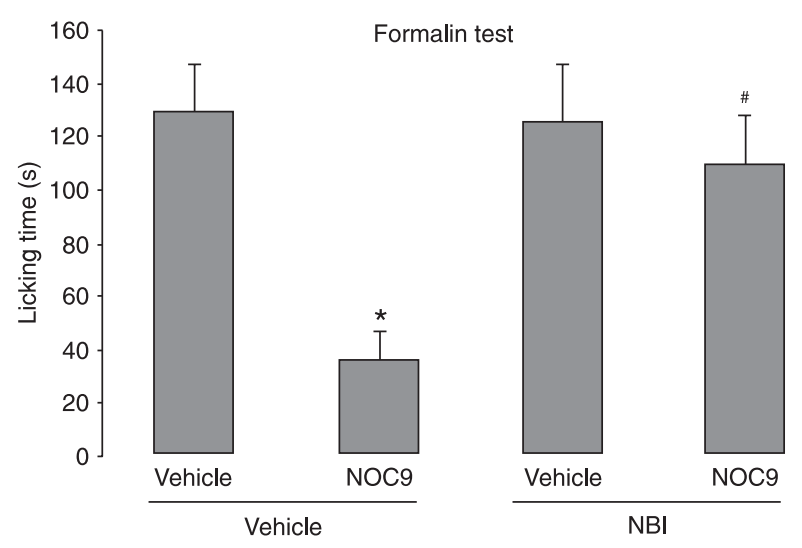

Figure 3. Effect of intra-dPAG microinjections of NBI 27914 (0 or $2 \mathrm{nmol}$ ) and NOC-9 (0 or $75 \mathrm{nmol}$ ) on the time (in seconds) spent licking the formalin affected paw in mice (nociceptive response). Mice received an intra-dPAG injection of NBI 27914 (pretreatment: 0 or $2 \mathrm{nmol} / 0.2 \mu \mathrm{L}$ ) and, $10 \mathrm{~min}$ later, an injection of NOC-9 (treatment: 0 or $75 \mathrm{nmol} / 0.2 \mu \mathrm{L}$ ) at the same midbrain site and were then tested immediately. Data are reported as means $\pm \mathrm{SEM}, \mathrm{N}=8$ per group. $\mathrm{dPAG}=$ dorsal periaqueductal gray; NBI 27914 = [5-chloro-4-(N-(cyclopropyl)methyl-Npropylamino)-2-methyl-6-(2,4,6-trichlorophenyl)aminopyridine]; NOC-9 = [6-(2-hydroxy-1-methyl-2-nitrosohydrazino)-N-methyl1-hexanamine]. ${ }^{*} \mathrm{P}<0.05$ compared to control group (vehicle + vehicle) and ${ }^{\#} \mathrm{P}<0.05$ compared to vehicle + NOC-9 group (twoway ANOVA followed by the Duncan post hoc test). (protected SAP: $\mathrm{F}_{1,38}=4.61, \mathrm{P}<0.05$; unprotected SAP: $\mathrm{F}_{1,38}=14.17, \mathrm{P}<0.05$; unprotected $\mathrm{HD}: \mathrm{F}_{1,38}=12.77, \mathrm{P}<$ 0.05; OAEE: $\left.F_{1,38}=9.22, P<0.05\right)$. Post hoc comparisons
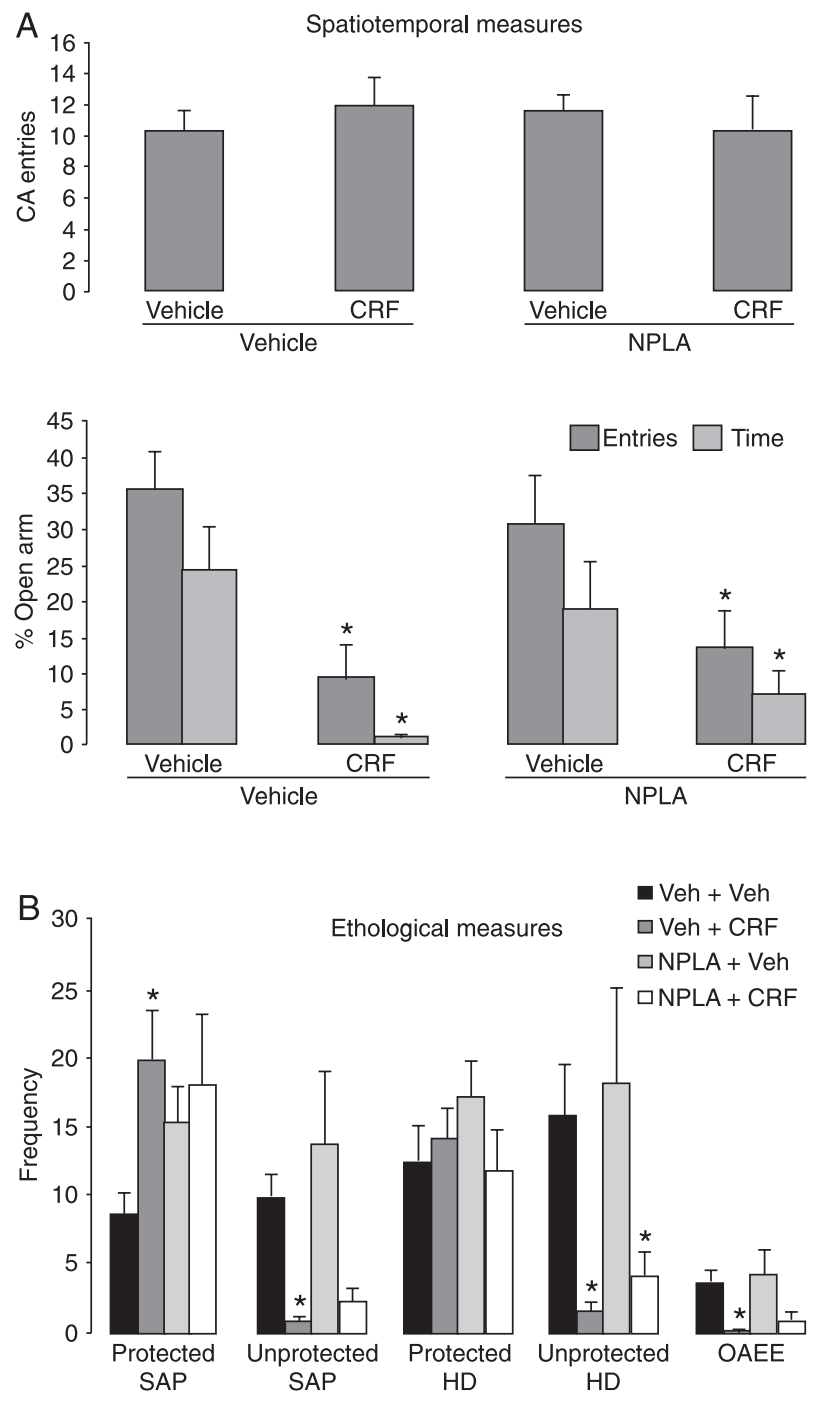

Figure 4. Effects of intra-dPAG microinjections of NPLA ( 0 or 0.4 $\mathrm{nmol}$ ) and CRF ( 0 or $150 \mathrm{pmol}$ ) on $(A)$ spatiotemporal measures: frequency of closed-arm (CA) entries (upper panel), percentage of open-arm entries and time (lower panel) and $(B)$ ethological measures: frequency of protected and unprotected stretchedattend posture (SAP), protected and unprotected head dipping (HD) and open-arm end exploration (OAEE) in mice exposed to the elevated plus-maze. Mice received intra-dPAG injection of NPLA (pretreatment: 0 or $0.4 \mathrm{nmol}$ ) and, 10 min later, CRF was injected (treatment: 0 or $150 \mathrm{pmol}$ ) into the same midbrain site. Testing started $10 \mathrm{~min}$ after the second injection. Data are reported as means \pm SEM, $\mathrm{N}=9-14$ per group. dPAG $=$ dorsal periaqueductal gray; NPLA $=N \omega$-propyl-L-arginine; CRF $=$ corticotropin-releasing factor. ${ }^{*} \mathrm{P}<0.05$ compared to control group (vehicle (Veh) + vehicle; two-way ANOVA followed by the Duncan post hoc test). 
indicated that previous treatment with NPLA was not able to reverse the CRF effect on these measures (protected SAP: $P=0.70$; unprotected SAP: $P=0.71$; unprotected HD: $P=0.64$; OAEE: $P=0.56)$. In addition, neither $C R F$ nor NPLA significantly changed the frequency of protected $\mathrm{HD}\left(\mathrm{F}_{1,38}=0.47, \mathrm{P}=0.49\right.$; Figure 4B).

Figure 5 shows the effects of combined injections of first NPLA ( 0 or $0.4 \mathrm{nmol}$ ) and then CRF (0 or $150 \mathrm{pmol})$ into the dPAG on time spent licking the paw affected by the $2.5 \%$ formalin injection. Two-way ANOVA did not reveal significant differences for the pretreatment (NPLA) factor $\left(F_{1,30}=\right.$ $0.91 ; P=0.34)$ or for pretreatment $x$ treatment interaction $\left(F_{1,30}=0.13 ; P=0.72\right)$, but did show a difference for the treatment $(\mathrm{CRF})$ factor $\left(\mathrm{F}_{1,30}=20.71 ; \mathrm{P}<0.05\right)$. Post hoc comparisons revealed that $\mathrm{CRF}$ (vehicle $+\mathrm{CRF}$ ) reduced the time spent licking the affected paw, compared to the control group (vehicle + vehicle), an effect that was not blocked by intra-dPAG pretreatment with NPLA.

\section{Discussion}

The results of the present study show that intra-dPAG injection of NOC-9 produces defensive-like behavior (running, jumping, and freezing) and inhibits the nociceptive response elicited during the formalin test in mice. Both defensive behavior and antinociception induced by this NO donor were attenuated by prior intra-dPAG injection of the CRFr1 antagonist NBI 27914. In contrast, the anxiogenic and antinociceptive effects produced by intra-dPAG injection of CRF were not changed by prior injection of the $\mathrm{nNOS}$ inhibitor NPLA into this midbrain site.

The defensive-like behavior induced by intra-dPAG NOC-9 in mice has been reported following intra-dPAG injection of this NO donor in rats (11). The cited investigators reported that intra-dPAG NOC-9 also reduced distance traveled. In agreement with those findings, the present study showed that intra-dPAG NOC-9 also reduced exploratory behavior, as indicated by a decrease in rearing frequency and locomotion time. Here, the decrease in locomotion and rearing following NOC-9 injection might have been a consequence of the increased freezing time. In other words, the exhibition of running, jumping and freezing induced by NOC-9 seemed to concur with the expression of exploratory behavior. Regarding the underlying mechanisms related to the aversive effects induced by the increase in NO synthesis, previous findings have emphasized the involvement of cGMP. Briefly, it has been shown that intra-PAG injection of ${ }^{1} \mathrm{H}-[1,2,4]$ oxadiazolol[4,3-a]quinoxalin-1-one] (ODQ), a selective soluble guanylyl cyclase (a cGMP synthesis enzyme) inhibitor, attenuates the aversive effects produced by local infusion of NOC-9 (11).

We have recently observed that intra-dPAG injection of CRF produces anxiety and antinociception in mice, both effects being completely blocked by prior local infusion of the CRFr1 antagonist, NBI 27914 (25). Importantly, the

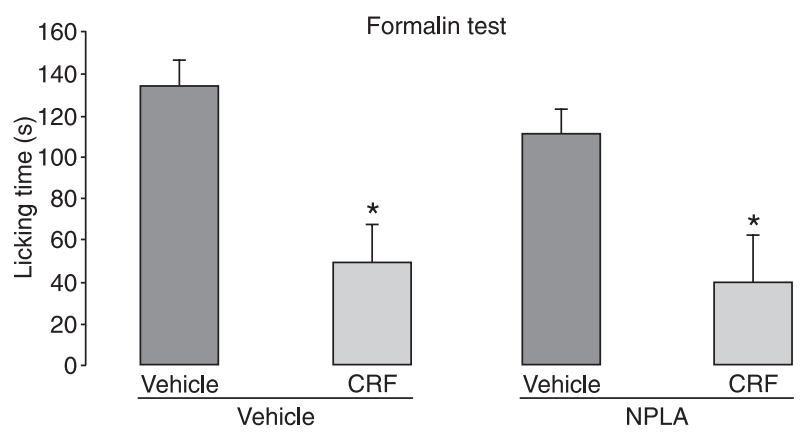

Figure 5. Effects of intra-dPAG microinjections of NPLA ( 0 or 0.4 $\mathrm{nmol}$ ) and CRF (0 or $150 \mathrm{pmol}$ ) on the time (in seconds) spent licking the formalin-treated paw. Mice received intra-dPAG injection of NPLA (pretreatment: 0 or $0.4 \mathrm{nmol}$ ) and, 10 min later, CRF was injected (treatment: 0 or $150 \mathrm{pmol}$ ) into the same midbrain site. Testing started $10 \mathrm{~min}$ after the second injection. Data are reported as means \pm SEM, $N=8$-11 per group. $d P A G=$ dorsal periaqueductal gray; NPLA $=\mathrm{N}^{\omega}$-propyl-L-arginine; CRF $=$ corticotropin-releasing factor. ${ }^{*} \mathrm{P}<0.05$ compared to control group (vehicle + vehicle; two-way ANOVA followed by the Duncan post hoc test).

anxiogenic and antinociceptive effects of CRF were not changed by intra-dPAG injection of antisauvagine-30 (25), a CRFr2 antagonist, suggesting that CRFr1 (but not CRFr2) located within the dPAG plays a role in the mediation of anxiety and pain inhibition in mice. Here, we investigated whether the blockade of CRFr1 would also attenuate the defensive behavior and antinociception observed with intra-dPAG injection of NOC-9. As shown in Figures 2 and 3, intra-dPAG injection of NBI 27914 prevented the behavioral and antinociceptive effects induced by the NO donor, suggesting that these aversive effects caused by an enhancement in NO synthesis are attenuated by CRFr1 blockade within the dPAG. The present results suggest that NO production may facilitate CRF release in this midbrain structure. This assumption is consistent with a study (16) demonstrating that CRF is released from cultures of amygdala and hypothalamus cells after incubation with nitroprusside, another type of NO donor. In addition, Lee et al. (26) have demonstrated that icv injection of 5-amino3-3(4-morpholinyl)-1,2,3-oxadiazolium chloride (SIN-1), also an NO donor, increases plasma ACTH, an effect that is abolished by prior treatment with anti-CRF antibody. How NO acts in favor of CRF release is unclear. However, Raber et al. (16) have suggested that NO may exert an excitatory effect directly upon the CRF neuron or indirectly via its action on neighboring glial cells. NO increase may, in turn, enhance intracellular calcium concentration, leading to exocytosis of CRF $(16,33)$. However, it is unlikely that the behavioral effects of intra-dPAG NO donor injection observed in the present study are exclusively related to an increase of CRF release within the dPAG, since intra-dPAG 
infusion of CRF does not induce jumping, running or freezing behavior $(21,25)$. Furthermore, it has been reported that NO also increases the release of other neurotransmitters, such as norepinephrine, acetylcholine and glutamate, in various brain areas (10). However, the present results suggest that CRFr1 plays an important role in the mediation of defensive behavior and antinociception induced by the enhancement in the nitrergic tonus within the midbrain dPAG of mice.

On the other hand, inhibition of NO synthesis within the PAG does not modify the anxiogenic and antinociceptive effects provoked by intra-dPAG injection of CRF, suggesting that $\mathrm{NO}$ does not play a pivotal role in these aversive effects of CRF. Corroborating previous studies in rats $(20,21)$ and mice (25), intra-dPAG CRF increased anxiety-like behavior in mice exposed to the EPM (Figure 5A), as well as provoking antinociception (25). Importantly, intra-dPAG injection of CRF did not change the number of closed-arm entries, a widely used measure of general activity in the EPM (see, e.g., Ref. 30), suggesting that this CRF-induced profile is behaviorally selective and thus not secondary to changes in general activity levels. Nevertheless, prior local infusion with the nNOS inhibitor, NPLA, neither changed anxiety indices in the EPM nor the antinociceptive effects induced by intradPAG CRF. We have observed that intra-PAG NPLA, at a dose similar to that used in the present study, completely blocked the defensive-like behavior and antinociception produced by intra-PAG injection of the glutamate NMDAr agonist $(1,27)$, suggesting that $0.4 \mathrm{nmol}$ NPLA is quite enough to attenuate these pro-aversive effects induced by NO production. In other words, NPLA did not change the effects of CRF, suggesting that NO production may not be involved in the CRFr-triggered intracellular cascade.

Bowers et al. (34) have shown that CRF has excitatory effects on neurons of the PAG in rats, an effect that has also been observed in other brain structures, such as locus coeruleus, hippocampus and cerebellum (35-37). The present results suggest that it is unlikely that CRF-induced anxiogenic and antinociceptive effects are mediated by NO

\section{References}

1. Miguel TT, Nunes-de-Souza RL. Defensive-like behaviors and antinociception induced by NMDA injection into the periaqueductal gray of mice depend on nitric oxide synthesis. Brain Res 2006; 1076: 42-48.

2. Schenberg LC, Povoa RM, Costa AL, Caldellas AV, Tufik S, Bittencourt AS. Functional specializations within the tectum defense systems of the rat. Neurosci Biobehav Rev 2005; 29: 1279-1298.

3. Blanchard DC, Hynd AL, Minke KA, Minemoto T, Blanchard RJ. Human defensive behaviors to threat scenarios show parallels to fear- and anxiety-related defense patterns of non-human mammals. Neurosci Biobehav Rev 2001; 25: 761-770.

4. Siegfried B, Frischknecht HR, Nunes de Souza RL. An etho- in the mouse PAG. These results contrast with the complete blockade of the aversive effects induced by NMDAr activation observed in animals pretreated with $\operatorname{NPLA}(1,27)$. Glutamate NMDAr activation leads to cellular calcium influx, which triggers a cascade of intracellular events including activation of NOS $(7,9)$, an NO synthesis enzyme. Although CRF also leads to cellular calcium influx, it is unlikely that the amount of this ion is enough to activate nNOS, since NPLA failed to block the anxiogenic and antinociceptive effects produced by intra-dPAG CRF. In other words, while the ionotropic NMDAr activation leads to an enhancement of intracellular calcium concentration, the stimulation of CRF receptors activates G-protein-coupled pathways, indicating two distinct mechanisms.

Furthermore, it is important to point out that CRF can induce glutamate release (38), so that the failure of NO inhibition to prevent the action of CRF does not rule out the possibility of glutamatergic mediation of the CRF anxiogenic and antinociceptive effects. This issue needs more clarification and further experiments, such as combined injection of CRF and glutamate receptor antagonists, need to be carried out in the future.

The results of the present study indicate that CRFr1 located within the dPAG plays an important role in the proaversive effects (defensive behavior and pain inhibition) induced by NO synthesis. In contrast, the anxiogenic and antinociceptive effects produced by the neuropeptide CRF do not depend on NO synthesis within this limbic midbrain structure in mice.

\section{Acknowledgments}

Research supported by FAPESP, CNPq (\#73102/20099), and PADC/FCF-UNESP. T.T. Miguel and K.S. Gomes were recipients of CAPES (\#AUX-PE-PNPD-2748/2010) and FAPESP (\#05/05171-1 and \#2010/01290-4) scholarships, respectively, and R.L. Nunes-de-Souza was the recipient of a CNPq (\#303580/2009-7) fellowship. logical model for the study of activation and interaction of pain, memory and defensive systems in the attacked mouse. Role of endogenous opioids. Neurosci Biobehav Rev 1990; 14: 481-490.

5. Engin $E$, Treit $D$. The effects of intra-cerebral drug infusions on animals' unconditioned fear reactions: a systematic review. Prog Neuropsychopharmacol Biol Psychiatry 2008; 32: 1399-1419.

6. Graeff FG. Serotonin, the periaqueductal gray and panic. Neurosci Biobehav Rev 2004; 28: 239-259.

7. Heresco-Levy U. Glutamatergic neurotransmission modulation and the mechanisms of antipsychotic atypicality. Prog Neuropsychopharmacol Biol Psychiatry 2003; 27: 11131123. 
8. Bandler R. Brain mechanisms of aggression as revealed by electrical and chemical stimulation: suggestion of a central role for the midbrain periaqueductal gray region. Vol. 13. In: Epstein A, Horrison A (Editors), Progress in psychobiology and physiological psychology. New York: Academic Press; 1988. p 67-154.

9. Garthwaite J, Garthwaite G, Palmer RM, Moncada S. NMDA receptor activation induces nitric oxide synthesis from arginine in rat brain slices. Eur J Pharmacol 1989; 172: 413416.

10. Prast $\mathrm{H}$, Philippu A. Nitric oxide as modulator of neuronal function. Prog Neurobiol 2001; 64: 51-68.

11. Braga AA, Aguiar DC, Guimaraes FS. NOC-9, a selective nitric oxide donor, induces flight reactions in the dorsolateral periaqueductal gray of rats by activating soluble guanylate cyclase. Neurosci Lett 2009; 459: 79-83.

12. Guimaraes FS, Beijamini V, Moreira FA, Aguiar DC, de Lucca AC. Role of nitric oxide in brain regions related to defensive reactions. Neurosci Biobehav Rev 2005; 29: 1313-1322.

13. Carvalho-Netto EF, Gomes KS, Amaral VC, Nunes-deSouza RL. Role of glutamate NMDA receptors and nitric oxide located within the periaqueductal gray on defensive behaviors in mice confronted by predator. Psychopharmacology 2009; 204: 617-625.

14. Leger L, Charnay Y, Burlet S, Gay N, Schaad N, Bouras C, et al. Comparative distribution of nitric oxide synthase- and serotonin-containing neurons in the raphe nuclei of four mammalian species. Histochem Cell Biol 1998; 110: 517525.

15. Onstott D, Mayer B, Beitz AJ. Nitric oxide synthase immunoreactive neurons anatomically define a longitudinal dorsolateral column within the midbrain periaqueductal gray of the rat: analysis using laser confocal microscopy. Brain Res 1993; 610: 317-324.

16. Raber J, Koob GF, Bloom FE. Interleukin-2 (IL-2) induces corticotropin-releasing factor (CRF) release from the amygdala and involves a nitric oxide-mediated signaling; comparison with the hypothalamic response. J Pharmacol Exp Ther 1995; 272: 815-824.

17. Albeck DS, McKittrick CR, Blanchard DC, Blanchard RJ, Nikulina J, McEwen BS, et al. Chronic social stress alters levels of corticotropin-releasing factor and arginine vasopressin mRNA in rat brain. J Neurosci 1997; 17: 4895-4903.

18. Blanchard DC, Sakai RR, McEwen B, Weiss SM, Blanchard RJ. Subordination stress: behavioral, brain, and neuroendocrine correlates. Behav Brain Res 1993; 58: 113-121.

19. Timpl P, Spanagel R, Sillaber I, Kresse A, Reul JM, Stalla $G K$, et al. Impaired stress response and reduced anxiety in mice lacking a functional corticotropin-releasing hormone receptor 1. Nat Genet 1998; 19: 162-166.

20. Britton DR, Koob GF, Rivier J, Vale W. Intraventricular corticotropin-releasing factor enhances behavioral effects of novelty. Life Sci 1982; 31: 363-367.

21. Martins AP, Marras RA, Guimaraes FS. Anxiogenic effect of corticotropin-releasing hormone in the dorsal periaqueductal grey. Neuroreport 1997; 8: 3601-3604.

22. Baldwin HA, Rassnick S, Rivier J, Koob GF, Britton KT. CRF antagonist reverses the "anxiogenic" response to ethanol withdrawal in the rat. Psychopharmacology 1991; 103: 227-
232.

23. Martins AP, Marras RA, Guimaraes FS. Anxiolytic effect of a $\mathrm{CRH}$ receptor antagonist in the dorsal periaqueductal gray. Depress Anxiety 2000; 12: 99-101.

24. Nishikawa H, Hata T, Itoh E, Funakami Y. A role for corticotropin-releasing factor in repeated cold stress-induced anxiety-like behavior during forced swimming and elevated plus-maze tests in mice. Biol Pharm Bull 2004; 27: 352356.

25. Miguel TT, Nunes-de-Souza RL. Anxiogenic and antinociceptive effects induced by corticotropin-releasing factor (CRF) injections into the periaqueductal gray are modulated by CRF1 receptor in mice. Horm Behav 2011; 60: 292-300.

26. Lee S, Kim CK, Rivier C. Nitric oxide stimulates ACTH secretion and the transcription of the genes encoding for NGFI-B, corticotropin-releasing factor, corticotropin-releasing factor receptor type 1 , and vasopressin in the hypothalamus of the intact rat. J Neurosci 1999; 19: 7640-7647.

27. Miguel TT, Nunes-de-Souza RL. Anxiogenic-like effects induced by NMDA receptor activation are prevented by inhibition of neuronal nitric oxide synthase in the periaqueductal gray in mice. Brain Res 2008; 1240: 39-46.

28. Paxinos G, Franklin KBJ. The mouse brain in stereotaxic coordinates. California: Academic Press; 2001.

29. Lister RG. The use of a plus-maze to measure anxiety in the mouse. Psychopharmacology 1987; 92: 180-185.

30. Rodgers RJ, Johnson NJ. Factor analysis of spatiotemporal and ethological measures in the murine elevated plus-maze test of anxiety. Pharmacol Biochem Behav 1995; 52: 297303.

31. Dubuisson D, Dennis SG. The formalin test: a quantitative study of the analgesic effects of morphine, meperidine, and brain stem stimulation in rats and cats. Pain 1977; 4: 161174.

32. Tjolsen A, Berge OG, Hunskaar S, Rosland JH, Hole K. The formalin test: an evaluation of the method. Pain 1992; 51: 5-17.

33. Nedergaard M. Direct signaling from astrocytes to neurons in cultures of mammalian brain cells. Science 1994; 263: 1768-1771.

34. Bowers LK, Swisher CB, Behbehani MM. Membrane and synaptic effects of corticotropin-releasing factor on periaqueductal gray neurons of the rat. Brain Res 2003; 981: 52-57.

35. Aldenhoff JB, Gruol DL, Rivier J, Vale W, Siggins GR. Corticotropin releasing factor decreases postburst hyperpolarizations and excites hippocampal neurons. Science 1983; 221: 875-877.

36. Fox EA, Gruol DL. Corticotropin-releasing factor suppresses the afterhyperpolarization in cerebellar Purkinje neurons. Neurosci Lett 1993; 149: 103-107.

37. Valentino RJ, Foote SL, Aston-Jones G. Corticotropinreleasing factor activates noradrenergic neurons of the locus coeruleus. Brain Res 1983; 270: 363-367.

38. Skorzewska A, Bidzinski A, Hamed A, Lehner M, Turzynska D, Sobolewska A, et al. The effect of CRF and alpha-helical $\operatorname{CRF}((9-41))$ on rat fear responses and amino acids release in the central nucleus of the amygdala. Neuropharmacology 2009; 57: 148-156. 